\title{
Birational geometry of surfaces
}

\author{
Ciro Ciliberto $^{1}$. Thomas Dedieu ${ }^{2}$. \\ Flaminio Flamini ${ }^{1}$. Rita Pardini $^{3}$
}

Published online: 12 March 2018

(C) Unione Matematica Italiana 2018

This volume is an issue of the Bollettino dell'Unione Matematica Italiana connected to the workshop "Birational geometry of surfaces" which took place at the Department of Mathematics of the University of Rome "Tor Vergata", Italy, in January, 11-15, 2016. We thank the Editors of the Bollettino dell'Unione Matematica Italiana for having accepted to dedicate this issue to this topic.

The workshop was organized by Ciro Ciliberto, Thomas Dedieu, Flaminio Flamini, Rita Pardini with the support of the projects:

- Geometria, Algebra e Combinatoria di Spazi di Moduli e Configurazioni-no. PRA2016-67—of the University of Pisa,

- National Research Project (PRIN 2010-11) Geometria delle Varietà Algebricheno. 2010S47ARA-005-(nodes of Pisa and Roma "Tor Vergata"),

- GDRE (CNRS-INdAM) GRIFGA 2012-2015,

- Families of subvarieties in complex algebraic varieties; this project has received funding from the European Union's Horizon 2020 research and innovation programme under the Marie Skłodowska-Curie grant agreement no. 652782.

The aim of the workshop was to bring together experts in the field of birational geometry of algebraic surfaces and of their moduli spaces to have a comparison of production in different research directions. There have been about 40 participants, both experts and young researchers in Algebraic Geometry, joining the workshop from all around the world.

The structure of the workshop was very dynamic. There have been a number of introductory talks in the mornings, whose goal was to stimulate further discussions, combined with some more advanced talks dedicated to more recent advances. In the afternoons there has been a division into working groups on problems, topics and issues chosen by the participants themselves.

$凶$ Ciro Ciliberto

cilibert@axp.mat.uniroma1.it

1 Università degli Studi di Roma Tor Vergata, 00133 Rome, Italy

2 Université Paul Sabatier, 31062 Toulouse, France

3 Università di Pisa, 56127 Pisa, Italy 
The workshop was very successful and this volume contains a short list of problems and questions, proposed and discussed during the workshop, together with some of the products of the working groups joint with more introductory expositions (all the papers underwent a rigorous refereeing process). Precisely, the contents are the following:

- Open Problems. This short text gathers problems and questions that have been proposed and discussed during the problem session held in the course of the workshop.

- F. Bastianelli, Irrationality issues for projective surfaces. This survey is concerned with various birational invariants extending the notion of "gonality" of curves to projective varieties of arbitrary dimension, and measuring the failure of a given projective variety to satisfy certain rationality properties, such as being uniruled, rationally connected, unirational, stably rational or rational. The author reviews a series of results describing these invariants for various classes of projective surfaces.

- F. Bastianelli, Corrigendum to the paper "Irrationality issues for projective surfaces". The author corrects statements of Theorem 3.3 and of Corollary 3.5 contained in his paper Irrationality issues for projective surfaces.

- F. Bastianelli, C. Ciliberto, F. Flamini, P. Supino, A note on gonality of curves on general hypersurfaces. The authors deal with the existence of curves with low gonality on smooth hypersurfaces $X \subset \mathbb{P}^{n+1}, n \geqslant 2$. After reviewing a series of results on this topic, they report on a recent progress they achieved as a product of the workshop namely: if $X \subset \mathbb{P}^{n+1}$ is a very general hypersurface of degree $d \geqslant 2 n+2$, the least gonality of a curve $C \subset X$ passing through a general point of $X$ is $\operatorname{gon}(C)=d-\left\lfloor\frac{\sqrt{16 n+1}-1}{2}\right\rfloor$, apart from a series of possible exceptions, where $\operatorname{gon}(C)$ may drop by one.

- J. Decaup, A. Dubouloz, Affine lines in the complement of a smooth plane conic. The authors classify closed curves isomorphic to the affine line in the complement of a smooth rational projective plane conic $Q$. Over a field of characteristic zero they show that, up to the action of the subgroup of the Cremona group of the plane consisting of birational endomorphisms restricting to biregular automorphisms outside $Q$, there are exactly two such lines: the restriction of a smooth conic osculating $Q$ at a rational point and the restriction of the tangent line to $Q$ at a rational point. In contrast, they give examples illustrating the fact that over fields of positive characteristic, there exist exotic closed embeddings of the affine line in the complement of $Q$. They also determine an explicit set of birational endomorphisms of the plane whose restrictions generate the automorphism group of the complement of $Q$ over a field of arbitrary characteristic.

- F. Favale, Calabi-Yau quotients with terminal singularities. The paper is concerned with quotients of Calabi-Yau threefolds with isolated singularities. More precisely, the author deals with the case of $X / G$ with terminal singularities and proves that in such a case, if $G$ is cyclic of prime order, then $G$ has order lower than or equal to 5 .

- C. Fontanari, D. Martinelli, Why should a birational geometer care about Bridgeland stability conditions? In this survey the authors borrow from Coskun and Huizenga an example of application of Bridgeland stability conditions to birational geometry and they rephrase it without assuming any previous knowledge about derived categories.

- M. Franciosi, S. Rollenske, Canonical rings of Gorenstein stable Godeaux surfaces. Extending the description of canonical rings from M. Reid (J. Fac. Sci. Univ. Tokyo Sect. I A Math. 25(1): 75-92, 1978), the authors show that every Gorenstein stable Godeaux surface with torsion of order at least 3 is smoothable.

- M. Lelli-Chiesa, Curves on surfaces with trivial canonical bundle. In this note the author surveys some results concerning Severi varieties and variation in moduli of curves lying 
on $K 3$ surfaces or on abelian surfaces. A number of open problems is listed and some work in progress is mentioned.

- F. Polizzi, Monodromy representations and surfaces with maximal Albanese dimension. In this paper, the author relates the existence of some surfaces of general type and maximal Albanese dimension to the existence of some monodromy representations of the braid group $B_{2}\left(C_{2}\right)$ in the symmetric group $S_{n}$. Furthermore, he computes the number of such representations up to $n=9$; for $n=2,3$ the author recovers some surfaces with $p_{g}=q=2$ recently studied (with different methods) by the author and his collaborators, whereas for $n=4$ he obtains some conjecturally new examples.

- S. Tirabassi, A note on the derived category of Enriques surfaces in characteristic 2. The goal of this paper is to show that the (twisted) derived category "recognizes" the three different kinds of Enriques surfaces in characteristic 2. 